\title{
The role of neuromodulation in chronic functional constipation: a systematic review
}

\author{
N. Pauwels ${ }^{1 \#}$, C. Willemse ${ }^{1 \#}$, S. Hellemans ${ }^{1 \#}$, N. Komen ${ }^{1,2}$, S. Van den Broeck ${ }^{1,2}$, J. Roenen ${ }^{1,3}$, T. Van Aggelpoel ${ }^{1,3}$, H. De \\ Schepper ${ }^{1,4}$ \\ \# Egal contribution. \\ (1) Faculty of Medicine and Health Sciences, University of Antwerp, Belgium ; (2) Department of Abdominal Surgery, University Hospital Antwerp, Belgium ; (3) \\ Department of Urology, University Hospital Antwerp, Edegem, Belgium ; (4) Department of Gastroenterology and Hepatology, University Hospital Antwerp, Belgium.
}

\begin{abstract}
Background: Chronic functional constipation is a highly prevalent disorder in which, when conservative measures fail to relieve symptoms, surgical interventions are sometimes indicated. In recent years, neuromodulation for the treatment of functional constipation has gained interest but its role and effectiveness are still unclear. The purpose of this review is to provide a systematic overview on the current literature on the different modalities of neurostimulation and their effect on chronic functional constipation in adults as reported in the literature.

Methods: A search in the literature for articles concerning the effect of different types of neuromodulation on constipation was performed in PubMed using extensive search terms for the different modalities of neuromodulation. Studies and trials were checked for eligibility. For all types of neuromodulation together, 27 articles were included.

Results: 17 studies were included on SNM (sacral nerve modulation). Although multiple studies show positive results on the effect of SNM in constipation, double-blind crossover RCT's (randomised controlled trials) showed no significant effect. 3 studies were included for tSNS (transcutaneous sacral nerve stimulation), 2 for PTNS (percutaneous tibial nerve stimulation) and 2 for TTNS (transcutaneous tibial nerve stimulation). Studies and trials on these modalities of neuromodulation reported ambiguous results on statistical significance of the effect. For transcutaneous IFC (interferential current therapy) 2 studies were included, which both reported a statistically significant effect on all outcomes.

Conclusion: The beneficial effect of neuromodulation in chronic functional constipation remains questionable. However, neuro-modulation might be worth considering in patients refractory to treatment before turning to more invasive measures. Future research should shed more light on the effects of neuromodulation in constipation. (Acta gastroenterol. belg., 2021, 84, 467-476).
\end{abstract}

Key words: Constipation, implantable neurostimulators, electric stimulation therapy, transcutaneous nerve stimulation, percutaneous electric nerve stimulation.

\section{Introduction}

Chronic constipation is a frequent disorder with a significant impact on quality of life (1). A systematic review and meta-analysis by Suares and Ford showed a pooled global prevalence of constipation of $14 \%$ (2). Constipation is more prevalent in women, with an overall pooled prevalence of $17.4 \%$ in women compared to $9.2 \%$ in men. The prevalence of constipation rises modestly with age (3).

Physicians used to define constipation based on the number of bowel movements ( $<3$ per week). Nowadays however, the definition of constipation is based on a number of dominant symptoms -such as a sense of incomplete evacuation, use of digital manoeuvres for evacuation of stool, excessive straining, failed or lengthy attempts to defecate, abdominal bloating and hard consistency of stools- as described by patients (4-9). As opposed to acute constipation (generally $<1$ week in duration), chronic constipation is defined as lasting for more than 3 months (4).

\section{Functional constipation}

Chronic constipation is divided in primary and secondary constipation. Secondary constipation can be caused by diet (low in fibre and fluid), drugs (e.g. diuretics, NSAIDs, opioids, antidepressants, etc.) metabolic disorders, neurological problems (Parkinson disease) or colorectal disease $(4,8,9)$. Primary (or functional) constipation results from disturbed regulation of stool propulsion, abnormal function of anorectal neuromuscular apparatus and disruption of the bidirectional pathways of the brain-gut axis $(4,5,8)$. Functional constipation is defined by the Rome IV criteria for functional constipation. This must include at least two of the following: straining during more than one-fourth of defecations, lumpy or hard stools (Bristol scale 1 or 2) more than one-fourth of defecations, sensation of incomplete evacuation more than one-fourth of defecations, sensation of anorectal obstruction more than one-fourth of defecations, manual manoeuvres to facilitate more than one-fourth of defecations and fewer than three spontaneous bowel movements per week. In addition, loose stools are rarely present without the use of laxatives and there are no sufficient criteria for IBS (irritable bowel syndrome). Primary constipation can be further categorized in three subtypes that largely overlap: normal transit constipation (with a substantial overlap with constipation-predominant irritable bowel syndrome or IBS-C), slow transit constipation and rectal evacuation disorders (4).

Correspondence to: Heiko De Schepper, MD-PhD, Department of Gastroenterology and Hepatology, University Hospital Antwerp, Wilrijkstraat 10, 2650 Edegem, Belgium. Phone : +32 (0)3 8215585 . Fax : +32 (0)3 8214478 . E-mail : heiko.deschepper@uza.be

Submission date : 17/01/2021

Acceptance date : 14/02/2021 


\section{Normal transit constipation}

The largest group of primary chronic constipation consists of NTC (normal transit constipation). These patients have no objective evidence of either slow transit constipation or a rectal evacuation disorder, yet do experience symptoms of chronic constipation (4). The pathophysiology of normal transit constipation is not entirely clear $(4,7)$.

\section{Slow transit constipation}

A second entity in functional constipation is STC (slow transit constipation). STC is most common in young women. Patients have decreased overall colonic motor activity, absent or impaired gastrocolonic response and increased retrograde propulsion (8). In severe STC, a loss of interstitial cells of Cajal (pacemaker cells of the gastrointestinal neuromuscular apparatus) was observed on pathological examination of resected colon (4).

\section{Rectal evacuation disorders}

The third category of functional constipation represents RED (rectal evacuation disorders). These are characterized by the inability to coordinate the abdominal and pelvic floor muscles to evacuate stools due to functional or structural (e.g. rectal prolapse) defects (4). The most common cause of rectal evacuation disorders is dyssynergic defecation (functional evacuation disorder), an acquired disorder in which defecation is hindered because of inadequate rectal and/or abdominal propulsive force, impaired anal relaxation or increased outlet resistance generated by paradoxical external anal sphincter or puborectalis muscle contraction $(4,7,8)$. For the Rome IV diagnosis of dyssynergic defecation, patients must meet 3 criteria: the occurrence of constipation symptoms, manometric or electromyographic evidence of a dyssynergic pattern during attempted defecation and one other abnormal colorectal test (e.g. balloon expulsion test or defecography) (1).

\section{Treatment}

Management of chronic constipation starts with withdrawal from potential predisposing drugs, education about constipation, lifestyle measures and ritualizing bowel habit. Lifestyle modifications consist of increasing fluid intake, fibre intake and physical activity (4). The main classes used in pharmacotherapy for chronic constipation are: osmotic laxatives, stimulant laxatives, secretagogues and serotonergic enterokinetic agents (4). An effective treatment for dyssynergic defecation is biofeedback therapy. The two main goals of biofeedback therapy are correction of dyssynergic defecation and improvement of rectal hyposensitivity.

In rectal evacuation disorder resistant to standard laxative therapy and biofeedback therapy, stimulant or water enemas can be applied to empty the distal colon. As a measure of last resort, surgical intervention may be considered (10): if a structural defecation disorder is present (e.g. a rectocele), restorative surgery is an option (e.g. a rectopexy). If there is associated slow transit constipation a colectomy with associated ileostomy is an option, when transit is normal, a colostomy should suffice (4). However, surgical intervention should only be reserved for carefully selected patients (11). Onlooking at the current treatment paradigm of chronic constipation, a treatment gap between the conservative treatment and surgical interventions is prominent. A rescue treatment is needed in order to prevent invasive surgical measures when patients fail on conservative treatment. The aim of this systematic review is to assess whether neuromodulation is a candidate to fill this treatment gap.

\section{Search method}

In October 2020, a search for articles concerning the effect of different types of neuromodulation on constipation was performed in PubMed. Only studies and trials investigating the effect of neuromodulation on functional constipation in adults were selected. Review articles and studies about the effect of neuromodulation in children were excluded. Also, studies regarding the effect of acupuncture were not included. Afterwards, studies that were considered were checked for eligibility by two authors independently. For sacral neuromodulation we used the search term: "((sacral nerve stimulation) $O R$ (sacral neuromodulation) OR (sacral neurostimulation)) AND (constipation)". This produced 240 results in PubMed of which in the end 17 studies concerning the effect of SNM in chronic constipation were deemed eligible if they met in- and exclusion criteria. Inclusion criteria were: studies and trials concerning the effect of the different modalities of neuromodulation on chronic functional constipation in adults. Trials studying the effect in children, review articles and articles in other languages than English, Dutch and French were excluded. For transcutaneous sacral nerve stimulation, the search term "((transcutaneous sacral nerve stimulation) $O R$ (tSNS) OR (transcutaneous sacral stimulation) OR (transcutaneous sacral neuromodulation) OR (TES) OR (TENS)) AND (constipation)" was used which rendered 137 results of which in the end only 3 were eligible. Studies on the effect of transcutaneous interferential current therapy were searched using "(interferential therapy) AND (constipation)" as search term, producing 29 results of which 2 were eligible. For percutaneous and transcutaneous tibial nerve stimulation the search terms "((percutaneous tibial nerve stimulation) OR (PTNS)) AND (constipation)" and "((transcutaneous tibial nerve stimulation) OR (TTNS)) AND (constipation)" were used respectively. 12 results, of which 2 suitable for inclusion, were produced for PTNS and 20 results were produced for TTNS, 3 of which were reckoned eligible. The studies included in this review are shown in the tables below. 


\section{Neuromodulation for chronic functional consti- pation}

There are multiple ways of achieving neuromodulation for constipation such as stimulation of the sacral plexus (i.e. sacral nerve modulation) and stimulation of the posterior tibial nerve either through transcutaneous or percutaneous electrodes. Both terms, neuromodulation and neurostimulation, are used interchangeably within the literature. We prefer to speak of neuromodulation rather than neurostimulation because SNM and other forms of neuromodulation have a possible effect on both faecal incontinence and constipation which suggests central neuromodulation (12).

\section{Sacral neuromodulation}

In sacral neuromodulation, an electrode is placed in the third sacral foramen and a neuromodulator is implanted subcutaneously in the gluteal region (12). Before implantation, the eligibility of patients can be tested using percutaneous nerve evaluation, a simple office-based screening test with high specificity and positive predictive value (13).

SNM was initially developed for the treatment of urinary dysfunction. Afterwards, additional benefits of this technique were discovered, including effects on bowel function (14). Nowadays, SNM is well established for the treatment of both urinary and faecal incontinence (15). Yet, SNM also appears to play a role in the treatment of conditions of impaired emptying, i.e. bladder voiding disorders and chronic constipation (16).

Initially, it was assumed that the effects of SNM occur as a result of direct peripheral motor neurostimulation. This was, however, not confirmed in recent studies, which showed that the effects of SNM occur at a pelvic afferent or central level (17). Because the therapy affects the neural control of both the urinary bladder and the bowel, it is suggested that SNM works through the same mechanism (16). Additionally, this 'single mechanism' hypothesis could explain the paradox that one treatment is effective in both faecal incontinence and chronic constipation (16-18). The exact mechanism of action, however, is complex and still remains largely unknown.

Despite studies reporting a good outcome of SNM in faecal incontinence, the treatment is not commonly used for constipation. Several double-blind crossover RCT's showed no significant effect of active stimulation compared to sham stimulation in refractory constipation $(19,20)$. As reported in a European consensus statement from Maeda et al., SNM for treatment of constipation has not been universally approved by regulatory authorities in Europe. Still, recent guidelines on functional constipation from the European society of Neurogastroenterology and Motility state that SNM may be worth trying in patients refractory to conservative treatment. Despite rather low success rates and low level of evidence, it should be considered due to its minimal invasive nature in comparison to other surgical interventions (21). An overview of the studies on the effect of SNM in functional constipation is shown in table 1.

\section{Transcutaneous sacral nerve stimulation}

tSNS is a potential non-invasive alternative for sacral neuromodulation (22). The mechanism of action is comparable to SNM and based on the activation of afferent neuronal networks. Using two pairs of electrodes placed on the skin over sacral nerves S1-S4, two independent types of direct current are simultaneously administered. Iqbal et al. investigated the improvement on the Wexner Constipation Score and improvement of quality of life after four weeks of therapy. However, the overall improvements in quality of life scores and weekly defecation frequency were not statistically significant.

Another comparative before-after study by Kim et al. did show a significant effect of tSNS on bowel movements and Constipation Assessment Scale (23) but also featured a small study population and a study design that is inherently susceptible to bias and lacks a control group.

In conclusion, there is no clear benefit of using tSNS for constipation. Evidence about the effect of tSNS on constipation is still scarce (24). Studies published on this topic are summarized in table 2.

\section{Transcutaneous interferential current therapy}

Another non-invasive technique, transcutaneous IFC, was invented to overcome a major dilemma concerning neuromodulation. In order to target the nerves of interest, the electric current has to pass the surrounding tissue and muscles. This can be achieved by using either a higher current, which can be painful, or a higher frequency. Unfortunately, these high-frequency currents are not suitable for nerve stimulation. IFC is based on the application of two slightly different medium-frequency alternating currents $( \pm 4000 \mathrm{~Hz})$ which produce a new modulated interferential current at their intersection point (25). IFC can be delivered transcutaneously by positioning four electrodes on the back or abdomen, or transabdominally with two electrodes on the back and two on the abdomen (22). All studies related to IFC and constipation in adults report a statistically significant improvement in symptom reduction but are hampered by weaknesses in study design and a challenging organization of an adequate sham treatment (22). An overview of the studies on the effect of IFC in functional constipation is shown in table 3.

\section{Tibial nerve stimulation}

A newer, cheaper and less invasive variant of SNM is TNS (tibial nerve stimulation). The tibial nerve is a branch of the sciatic nerve. It is a mixed nerve containing both motor and sensory fibres originating 


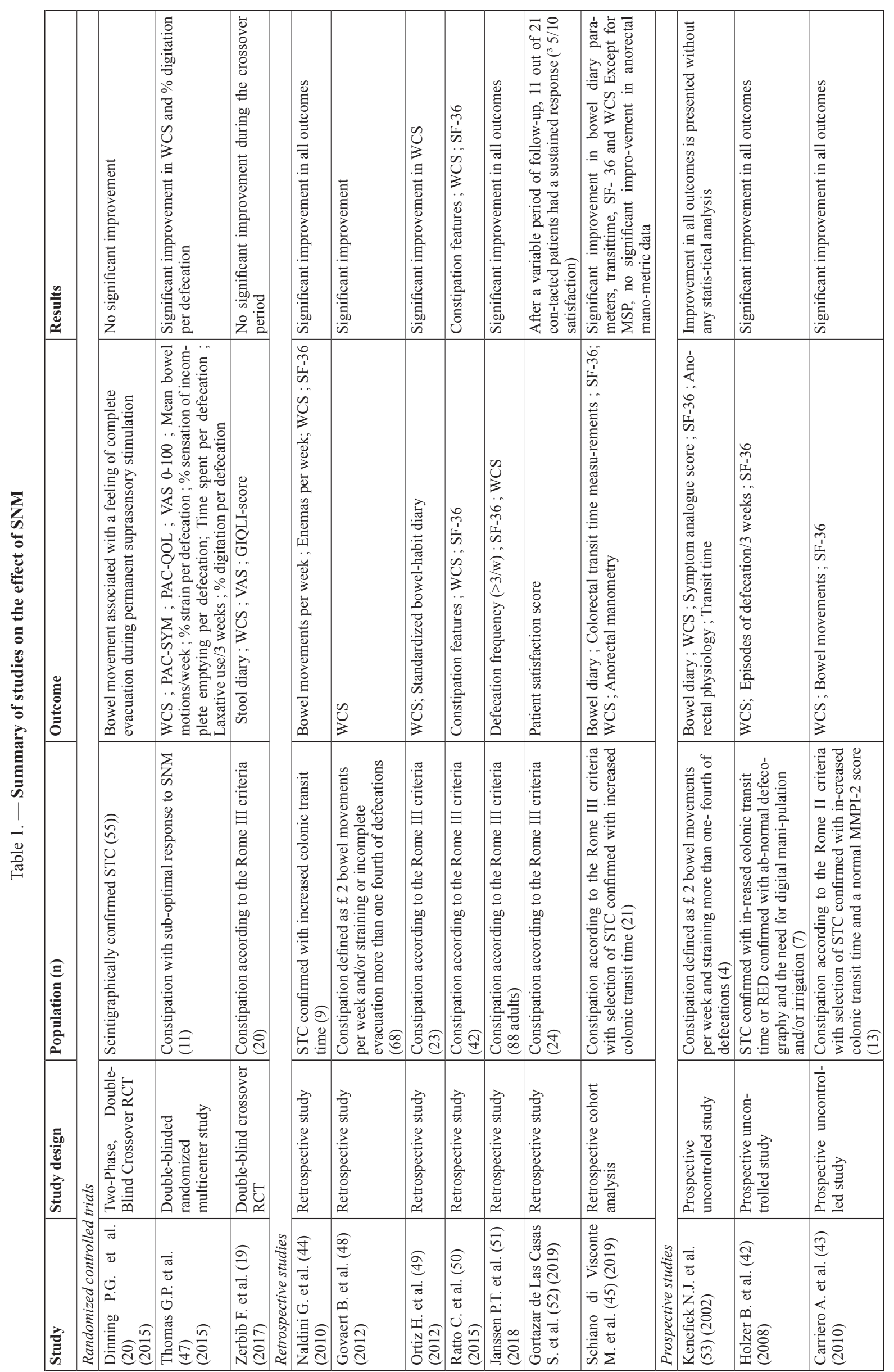



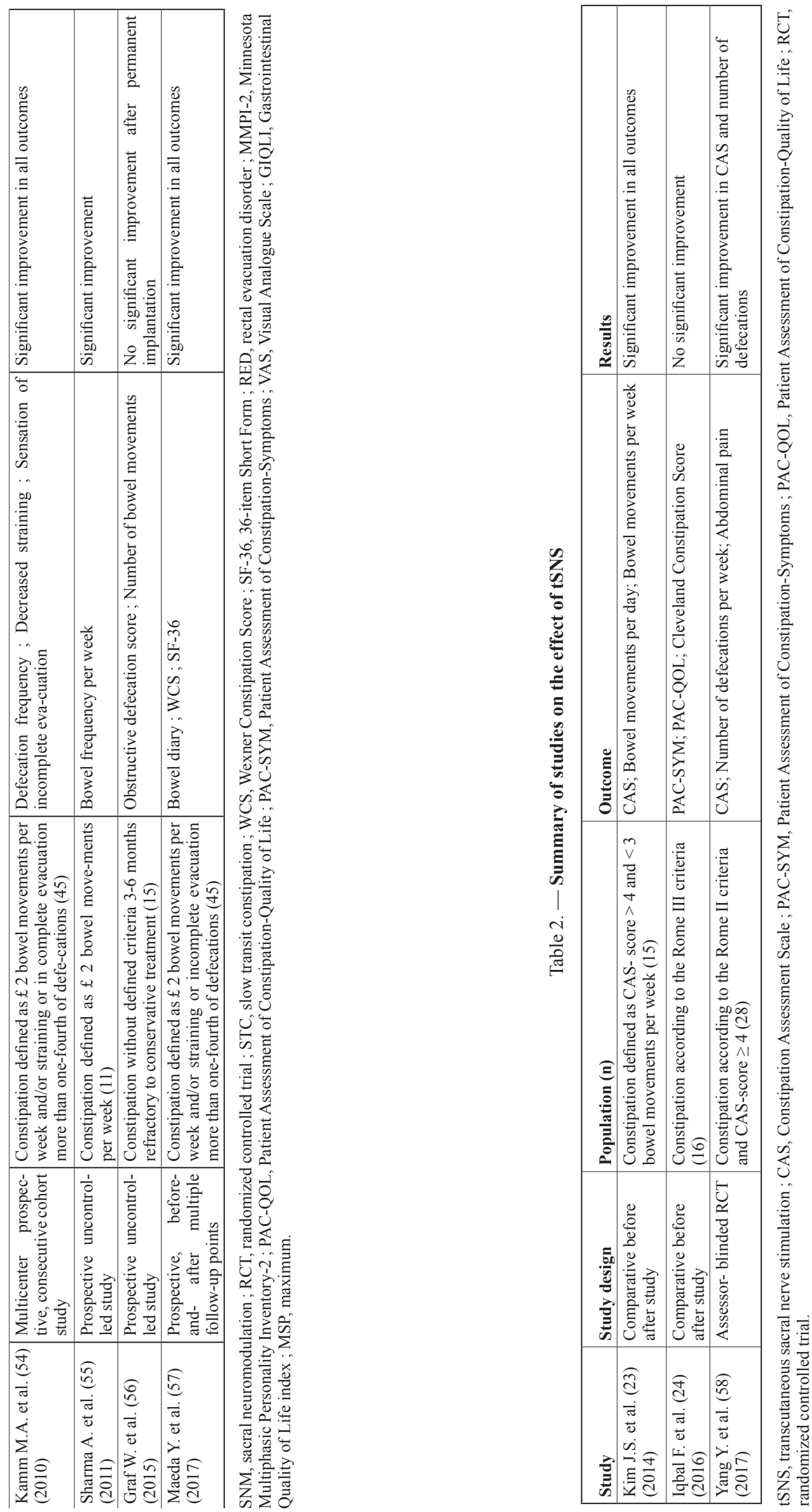

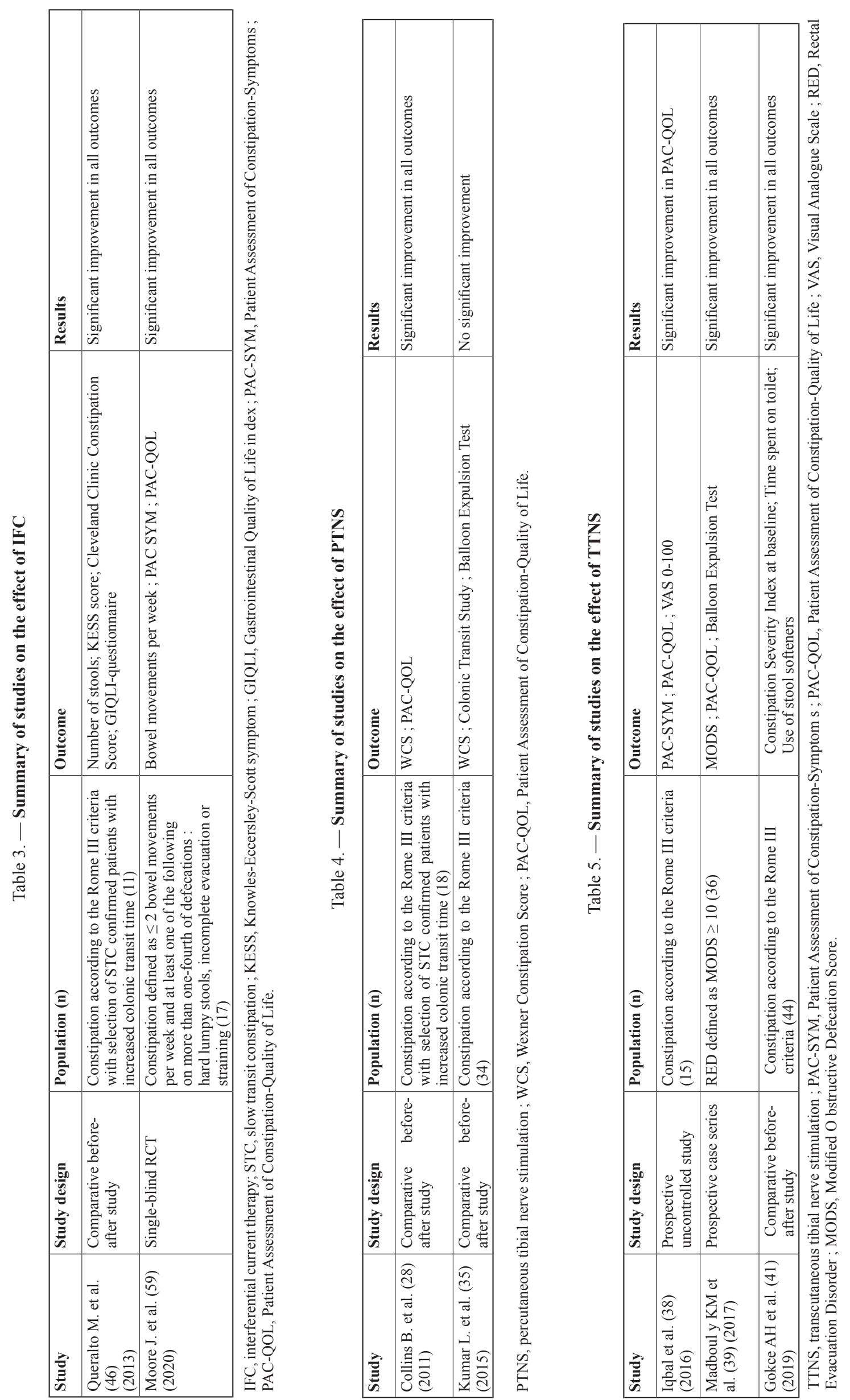
from the L4-S3 roots of the sacral plexus. These roots provide stimulation to the genitourinary tract as well as the pelvic floor muscles and the lower gastrointestinal tract. Although the exact mechanism of neurostimulation is unclear, the mechanism of action can be viewed as one of three possibilities: central, autonomic and/ or enteric mechanism (26). It is believed that TNS involves specific receptors in the spinal cord (27), neuromodulates bowel motility and/or sphincter function by transmitting impulses to the sacral nerves (28) and induces neural plasticity in the central nervous system (29). Stimulation of the tibial nerve is thought to lead to similar changes in anorectal neuromuscular function as SNM because of shared sacral segmental innervation (30). Nevertheless, there are some differences between these two interventions. TNS, in contrast to SNM, does not use a continuous electric stimulation to obtain clinical improvement (29). Although SNM is a safe treatment, it is a more invasive intervention with more adverse events such as wound infection and lead migration with surgical revisions required in $28,8 \%$ of patients $(31,32)$. Adverse events associated with TNS are relatively uncommon, but include bruising, bleeding, tingling and mild pain. Due to its non-invasive character, no major adverse events are described. Finally, the cost of SNM is considerably higher than TNS (33).

Two variants of TNS were reviewed: PTNS and TTNS.

\section{PTNS}

Percutaneous tibial nerve stimulation, developed by Stoller et al. in 1987, has gained an important place in the treatment of faecal incontinence (34). Yet, its effectiveness in managing constipation has only been discussed in two published studies $(28,35)$. The commonly accepted protocol describes the application of a 34-Gauge needle electrode $5 \mathrm{~cm}$ cephalad to the medial malleolus and $2 \mathrm{~cm}$ posterior to the tibia. The needle is inserted approximately $2 \mathrm{~cm}$ into the leg and the electrode is connected to the lead wire. The superficial electrode is attached near the medial aspect of the calcaneus. The right strength of current can be determined by slowly increasing the current until a response is provoked. This response can be either motor (flexion of the toe) or sensory (tingling in the heel or toes). Once the response is obtained, the current level is reduced slightly to subthreshold $(28,35)$. Most stimulators use a fixed pulse frequency of $20 \mathrm{~Hz}$ and pulse width of $200 \mu$ s. Previous studies have suggested that changing these parameters may affect success rate in treating constipation (35). The few studies concerning the effect of PTNS in chronic constipation have used the same treatment protocol that is widely applied for the treatment overactive bladder and faecal incontinence. This consists of 30 minutes of PTNS weekly for 12 weeks (35). It has been suggested that PTNS is more effective than methods using surface electrodes for stimulating the tibial nerve, probably because the needle electrode is closer to the tibial nerve (36). Whereas
Collins et al. observed a significant improvement in Wexner Constipation Score, no significant improvement in Wexner Constipation Score was observed by Kumar et al. in a similar study $(28,35)$. Both studies, being comparative before-after trials, don't feature a control group and have a rather small population size. In order to reach more conclusive statements about the effect of PTNS on functional constipation, studies featuring larger study populations investigating the long-term efficacy of PTNS are needed (37). Current studies on the effect of PTNS on functional constipation are summarized in table 4.

\section{TTNS}

As with PTNS, transcutaneous tibial nerve stimulation also targets the posterior tibial nerve but without requiring needle punctures. Adhesive surface stimulation electrodes are attached to the skin. The positive electrode is placed $10 \mathrm{~cm}$ cranially to the medial malleolus. A second, negative, electrode is placed behind the medial malleolus on the same leg $(38,39)$. There is no consensus regarding the fact that stimulation should be performed unilaterally or bilaterally. However, a pilot study on bilateral TTNS predicts a better efficacy in comparison with unilateral stimulation, as is the case with other types of neurostimulation (40). Since TTNS requires no needle punctures, it is a treatment that can be safely used by patients in their home environment (38).

There is a lack of standardized TTNS treatment protocols and knowledge about the optimal TTNS parameters, especially for constipation. Only few studies about the effect of TTNS on constipation have been published. These studies all feature a small and heterogeneous study population, use different stimulation parameters and different outcome measures. Stimulation parameters vary with a frequency ranging from $10-14 \mathrm{~Hz}$ and a pulse width ranging from 200-220 $\mu$ s. The amperage is determined in the same way as PTNS. Also, factors predicting response to TTNS have not yet been identified. Therefore, a mechanistic study with a larger population is required for determining the efficacy of TTNS in functional constipation (37).

In recent years, a couple of studies were performed on the effect of TTNS in chronic constipation. These are shown in table 5 . Iqbal et al. treated 15 patients with chronic constipation with 30 minutes of daily bilateral TTNS for 6 weeks straight. Clinical improvement, defined as a PAC-QoL score difference of at least one point, was seen in four patients $(26 \%)$. Only two patients showed clinical improvement on the PAC-SYM score (38).

Madbouly et al. applied TTNS to patients with proven rectal evacuation disorder without anatomic obstruction who had failed on conservative treatment. 36 patients received TTNS sessions of 30 minutes, three times per week for six weeks. 17 patients (47\%) stopped the use of any method to assist defecation in 
$>75 \%$ of their defecation attempts and were considered as responders (39). Most recently, Gokce et al. treated 44 geriatric patients aged $>65$ years with bilateral TTNS. For 6 weeks, patients were administered sessions of 30 minutes, three times per week. While 28 patients $(63,6 \%)$ were using stool softeners at baseline, only 7 patients $(15,9 \%)$ were using stool softeners after six weeks of treatment (41). It is remarkable that these studies mostly use different outcome measures. Here as well, further research in the form of blinded randomized controlled trials and larger sample sizes is required to draw more definite conclusions about the effect of TTNS in chronic functional constipation.

\section{Effect of neuromodulation on different subtypes of functional constipation}

Of the 17 trials included concerning SNM in functional constipation only 5 mentioned the further categorization of their population into the different subtypes of functional constipation. One trial studied the effect of SNM in both patients with STC and patients with RED (42). In this trial there is a significant improvement in all outcomes for both subtypes, yet no comparison was made between STC and RED. 4 trials only concerned patients with slow transit constipation (20,43-45). Two of which showed a significant improvement in all outcomes (43, 44). One study showed a significant improvement in all outcomes but anorectal manometric data (45). The fourth trial, a two-phase double-blind crossover RCT by Dinning et al. did not show a significant improvement in any outcome (20). However, this study used 'feeling of complete evacuation' after bowel movement as an outcome measure as compared to the other trials which consistently assessed the effects of SNM based upon more objective outcome measures (Wexner Constipation Score, 36-Item Short Form Health Survey and number of bowel movements). None of the included studies on SNM explicitly featured a population of patients with normal transit constipation. Thus, studies that focus on the subgroup of patients with slow transit constipation tend to show a significant effect on functional constipation. However, due to the lack of studies concerning the other subtypes of functional constipation, it is not possible to state that SNM is more effective in patients with STC as compared to NTC and RED.

None of the trials included for tSNS mentions further subdivision of their population based on the subtypes of functional constipation. Both for IFC and PTNS one article is included that studies the effect of these types of neuromodulation in STC $(28,46)$. For TTNS one article was included that evaluates the effect in patients with RED (39). Despite these three studies reporting a significant improvement in all outcomes, no valuable statement can be made on the effect of neuromodulation techniques on the different subtypes of functional constipation given the shortage of available literature that focus on these subtypes.

\section{Conclusion}

To date, multiple modalities of neuromodulation for the treatment of chronic constipation are available. Neuromodulation might be a rescue therapy, possibly able to fill the treatment gap that currently exists after conservative treatment fails and before surgical therapy is considered. Nevertheless, to date the beneficial effect of neuromodulation for chronic constipation remains questionable. In this literature review we have discussed the most widely studied types of neuromodulation. Sacral neuromodulation, a well-established treatment for urinary and faecal incontinence, is not commonly used in chronic constipation. Multiple double-blind randomized controlled trials have shown no significant effect in comparison with sham therapy. Still, guidelines have stated that due to its minimal invasive nature it might be worth trying in patients refractory to conservative treatment before turning to more invasive surgical interventions. A transcutaneous variant of sacral neuromodulation, transcutaneous sacral nerve stimulation, also has shown no significant benefit. A more complex alternative, transcutaneous interferential current therapy, did show a statistically significant beneficial effect in chronic constipation. Neurostimulation of the tibial nerve, either percutaneous through needle electrodes or transcutaneous through adhesive electrodes, also has shown no unambiguous effect in chronic constipation. So far, only rather low powered studies with a small sample size have investigated the role of percutaneous and transcutaneous tibial nerve stimulation in chronic constipation. To conclude, more research on the topic of neuromodulation in chronic constipation is required. Future research should aim at a thorough optimization and standardization of stimulation parameters as well as strengthening of study design by consistently using sham, randomization and blinding, while simultaneously using larger sample sizes. Whilst most of the current literature on neuromodulation in chronic functional constipation has shown no significant improvement of constipation related symptoms and quality of life, more powerful studies might uncover a subgroup of patients with chronic functional constipation in whom these therapies can be beneficial. Thus, we can conclude that at this point the use of different modalities of neuromodulation for chronic functional constipation can neither be rejected nor encouraged. Therefore, it can be stated that for now neuromodulation has not been able to fill the existing treatment gap in the treatment of chronic functional constipation. It is certain, however, that this will not have been the last debate regarding the role of neuromodulation in chronic functional constipation.

\section{Author contributions}

All three first authors (NP, CW and $\mathrm{SH}$ ) contributed to performing the literature search, reviewing the studies that were included and writing the manuscript. 
HDS conceived the idea for the review and supervised all stages in making the review. NK, SVDB, JR and TVA revised the article and gave instructions for final adjustments. All authors approved the final draft before applying for publication.

\section{Conflict of interest}

None of the authors received any financial support whatsoever, neither was there any conflict of interest.

\section{References}

1. RAO S.S., PATCHARATRAKUL T. Diagnosis and Treatment of Dyssynergic Defecation. Journal of neurogastroenterology and motility, 2016, 22(3) : 42335 .

2. SUARES N.C., FORD A.C. Prevalence of, and risk factors for, chronic idiopathic constipation in the community : systematic review and metaanalysis. The American journal of gastroenterology, 2011, 106(9) : 1582-91; quiz $1,92$.

3. HIGGINS P.D., JOHANSON J.F. Epidemiology of constipation in North America: a systematic review. The American journal of gastroenterology, 2004, 99(4) : 750-9.

4. CAMILLERI M., FORD A.C., MAWE G.M., DINNING P.G., RAO S.S., CHEY W.D., et al. Chronic constipation. Nature reviews Disease primers, 2017, $3: 17095$.

5. SHARMA A., RAO S. Constipation: Pathophysiology and Current Therapeutic Approaches. Handbook of experimental pharmacology, 2017, 239 : 59-74.

6. ANDROMANAKOS N.P., PINIS S.I., KOSTAKIS A.I. Chronic severe constipation: current pathophysiological aspects, new diagnostic approaches, and therapeutic options. European journal of gastroenterology \& hepatology, 2015, 27(3) : 204-14

7. ANDREWS C.N., STORR M. The pathophysiology of chronic constipation. Canadian journal of gastroenterology, 2011, 25 Suppl B : 16B-21B.

8. RAO S.S., RATTANAKOVIT K., PATCHARATRAKUL T. Diagnosis and management of chronic constipation in adults. Nature reviews Gastroenterology \& hepatology, 2016, 13(5) : 295-305.

9. COSTILLA V.C., FOXX-ORENSTEIN A.E. Constipation: understanding mechanisms and management. Clinics in geriatric medicine, 2014, 30(1): 107-15.

10. THAHA M.A., ABUKARA.A., THIN N.N., RAMSANAHIE A., KNOWLES C.H. Sacral nerve stimulation for faecal incontinence and constipation in adults. The Cochrane database of systematic reviews, 2015, (8) : Cd004464.

11. IQBAL F., VAN DER PLOEG V., ADABA F., ASKARI A., MURPHY J., NICHOLLS R.J., et al. Patient-Reported Outcome After Ostomy Surgery for Chronic Constipation. J Wound Ostomy Continence Nurs, 2018, 45(4) : 31925.

12. VAN WUNNIK B.P., BAETEN C.G., SOUTHWELL B.R. Neuromodulation for constipation: sacral and transcutaneous stimulation. Best practice \& research Clinical gastroenterology, 2011, 25(1) : 181-91.

13. BANAKHAR M., HASSOUNA M. Percutaneous Nerve Evaluation Test Versus Staged Test Trials for Sacral Neuromodulation: Sensitivity, Specificity, and Predictive Values of Each Technique. Int. Neurourol. J., 2016, 20(3) : 250-4.

14. NORDENSTAM J., BOLLER A.M., MELLGREN A. Sacral Nerve Stimulation in the Treatment of Bowel Disorders. Progress in neurological surgery, 2015, $29: 200-12$

15. PILKINGTON S.A., EMMETT C., KNOWLES C.H., MASON J., YIANNAKOU Y. Surgery for constipation: systematic review and practice recommendations: Results V: Sacral Nerve Stimulation. Colorectal disease : the official journal of the Association of Coloproctology of Great Britain and Ireland, 2017, 19 Suppl $3: 92-100$

16. JONES J., VAN DE PUTTE D., DE RIDDER D., KNOWLES C., O'CONNELL R., NELSON D., et al. A Joint Mechanism of Action for Sacral Neuromodulation for Bladder and Bowel Dysfunction? Urology, 2016, 97 : 13-9.

17. CARRINGTON E.V., EVERS J., GROSSI U., DINNING P.G., SCOTT S.M., O'CONNELL P.R., et al. A systematic review of sacral nerve stimulation mechanisms in the treatment of fecal incontinence and constipation. Neurogastroenterology and motility : the official journal of the European Gastrointestinal Motility Society, 2014, 26(9) : 1222-37.
18. COHN J.A., KOWALIK C.G., KAUFMAN M.R., REYNOLDS W.S., MILAM D.F., DMOCHOWSKI R.R. Evaluation of the axonics modulation technologies sacral neuromodulation system for the treatment of urinary and fecal dysfunction. Expert review of medical devices, 2017, 14(1) : 3-14.

19. ZERBIB F., SIPROUDHIS L., LEHUR P.A., GERMAIN C., MION F., LEROI A.M., et al. Randomized clinical trial of sacral nerve stimulation for refractory constipation. Br. J. Surg., 2017, 104(3) : 205-13.

20. DINNING P.G., HUNT L., PATTON V., ZHANG T., SZCZESNIAK M., GEBSKI V., et al. Treatment efficacy of sacral nerve stimulation in slow transit constipation: a two-phase, double-blind randomized controlled crossover study. The American journal of gastroenterology, 2015, 110(5) : 733-40.

21. SERRA J., POHL D., AZPIROZ F., CHIARIONI G., DUCROTTÉ P., GOURCEROL G., et al. European society of neurogastroenterology and motility guidelines on functional constipation in adults. Neurogastroenterology and motility : the official journal of the European Gastrointestinal Motility Society, 2020, 32(2) : e13762.

22. SOUTHWELL B.R. Electro-Neuromodulation for Colonic Disorders-Review of Meta-Analyses, Systematic Reviews, and RCTs. Neuromodulation, 2020.

23. KIM J.S., YI S.J. Effects of Low-frequency Current Sacral Dermatome Stimulation on Idiopathic Slow Transit Constipation. J. Phys. Ther. Sci., 2014, 26(6) : 831-2.

24. IQBAL F., THOMAS G.P., TAN E., ASKARI A., DASTUR J.K., NICHOLLS J., et al. Transcutaneous Sacral Electrical Stimulation for Chronic Functional Constipation. Dis. Colon. Rectum, 2016, 59(2) : 132-9.

25. MOORE J.S., GIBSON P.R., BURGELL R.E. Neuromodulation via Interferential Electrical Stimulation as a Novel Therapy in Gastrointestinal Motility Disorders. Journal of neurogastroenterology and motility, 2018, 24(1) : 19-29.

26. ABELl T.L., CHEN J., EMMANUEL A., JOLLEY C., SARELA A.I., TORNBLOM H. Neurostimulation of the gastrointestinal tract : review of recent developments. Neuromodulation, 2015, 18(3) : 221-7; discussion 7.

27. VAN DER PAL F., HEESAKKERS J.P., BEMELMANS B.L. Current opinion on the working mechanisms of neuromodulation in the treatment of lower urinary tract dysfunction. Current opinion in urology, 2006, 16(4) : 261-7.

28. COLLINS B., NORTON C., MAEDA Y. Percutaneous tibial nerve stimulation for slow transit constipation: a pilot study. Colorectal disease : the official journal of the Association of Coloproctology of Great Britain and Ireland, 2012, 14(4) : e165-70.

29. FINAZZI-AGRO E., ROCCHI C., PACHATZ C., PETTA F., SPERA E., MORI F., et al. Percutaneous tibial nerve stimulation produces effects on brain activity: study on the modifications of the long latency somatosensory evoked potentials. Neurourology and urodynamics, 2009, 28(4) : 320-4.

30. KNOWLES C.H., HORROCKS E.J., BREMNER S.A., STEVENS N., NORTON C., O'CONNELL P.R., et al. Percutaneous tibial nerve stimulation versus sham electrical stimulation for the treatment of faecal incontinence in adults (CONFIDeNT): a double-blind, multicentre, pragmatic, parallel-group, randomised controlled trial. Lancet (London, England), 2015, 386(10004) : 1640-8.

31. SIMILLIS C., LAL N., QIU S., KONTOVOUNISIOS C., RASHEED S., TAN E., et al. Sacral nerve stimulation versus percutaneous tibial nerve stimulation for faecal incontinence: a systematic review and meta-analysis. International journal of colorectal disease, 2018, 33(5) : 645-8.

32. ZEITON M., FAILY S., NICHOLSON J., TELFORD K., SHARMA A. Sacral nerve stimulation-hidden costs (uncovered). International journal of colorectal disease, 2016, 31(5) : 1005-10.

33. STASKIN D.R., PETERS K.M., MACDIARMID S., SHORE N., DE GROAT W.C. Percutaneous tibial nerve stimulation: a clinically and cost effective addition to the overactive bladder algorithm of care. Curr. Urol. Rep., 2012, 13(5) : 327-34.

34. STOLLER M.L., COPELAND S., MILLARD R.J., MURNAGHAN G.F. The Efficacy of Acupuncture in Reversing the Unstable Bladder in Pig-Tailed Monkeys. Journal of Urology, 1987, 137(6) : 104A-A.

35. KUMAR L., LIWANAG J., ATHANASAKOS E., RAEBURN A., ZARATELOPEZ N., EMMANUEL A.V. Effectiveness of percutaneous tibial nerve stimulation in managing refractory constipation. Colorectal disease : the official journal of the Association of Coloproctology of Great Britain and Ireland, 2017, 19(1) : 45-9.

36. GOVAERT B., PARES D., DELGADO-AROS S., LA TORRE F., VAN GEMERT W.G., BAETEN C.G. A prospective multicentre study to investigate percutaneous tibial nerve stimulation for the treatment of faecal incontinence. Colorectal disease : the official journal of the Association of Coloproctology of Great Britain and Ireland, 2010, 12(12) : 1236-41.

37. GEORGE A.T., MAITRA R.K., MAXWELL-ARMSTRONG C. Posterior tibial nerve stimulation for fecal incontinence: where are we? World journal of gastroenterology, 2013, 19(48) : 9139-45. 
38. IQBAL F., COLLINS B., THOMAS G.P., ASKARI A., TAN E., NICHOLLS R.J., et al. Bilateral transcutaneous tibial nerve stimulation for chronic constipation. Colorectal disease : the official journal of the Association of Coloproctology of Great Britain and Ireland, 2016, 18(2) : 173-8.

39. MADBOULY K.M., ABBAS K.S., EMANUEL E. Bilateral Posterior Tibial Nerve Stimulation in the Treatment of Rectal Evacuation Disorde : A Preliminary Report. Dis. Colon Rectum, 2017, 60(3) : 311-7.

40. THOMAS G.P., DUDDING T.C., NICHOLLS R.J., VAIZEY C.J. Bilateral transcutaneous posterior tibial nerve stimulation for the treatment of fecal incontinence. Dis. Colon Rectum, 2013, 56(9) : 1075-9.

41. GOKCE A.H., GOKCE F.S. Effects of bilateral transcutaneous tibial nerve stimulation on constipation severity in geriatric patients: A prospective clinical study. Geriatr. Gerontol. Int., 2020, 20(2) : 101-5.

42. HOLZER B., ROSEN H.R., NOVI G., AUSCH C., HÖLBLING N., HOFMANN M., et al. Sacral nerve stimulation in patients with severe constipation. Dis. Colon Rectum, 2008, 51(5) : 524-29; discussion 9-30.

43. CARRIERO A., MARTELLUCCI J., TALENTO P., FERRARI C.A. Sacral nerve stimulation for constipation: do we still miss something? Role of psychological evaluation. International journal of colorectal disease, 2010, 25(8) : 1005-10.

44. NALDINI G., MARTELLUCCI J, MORALDI L., BALESTRI R., ROSSI M. Treatment of slow-transit constipation with sacral nerve modulation. Colorectal disease : the official journal of the Association of Coloproctology of Great Britain and Ireland, 2010, 12(11) : 1149-52.

45. SCHIANO DI VISCONTE M., PASQUALI A., CIPOLAT MIS T., BRUSCIANO L., DOCIMO L., BELLIO G. Sacral nerve stimulation in slow-transit constipation : effectiveness at 5-year follow-up. International journal of colorectal disease, 2019, 34(9) : 1529-40.

46. QUERALTO M., VITTON V., BOUVIER M., ABYSIQUE A., PORTIER G. Interferential therapy: a new treatment for slow transit constipation. a pilot study in adults. Colorectal disease : the official journal of the Association of Coloproctology of Great Britain and Ireland, 2013, 15(1) : e35-9.

47. THOMAS G.P., DUELUND-JAKOBSEN J., DUDDING T.C., BRADSHAW E., NICHOLLS R.J., ALAM A., et al. A double-blinded randomized multicentre study to investigate the effect of changes in stimulation parameters on sacral nerve stimulation for constipation. Colorectal disease : the official journal of the Association of Coloproctology of Great Britain and Ireland, 2015, 17(11) : 990-5.

48. GOVAERT B., MAEDA Y., ALBERGA J., BUNTZEN S., LAURBERG S., BAETEN C.G. Medium-term outcome of sacral nerve modulation for constipation. Dis. Colon Rectum, 2012, 55(1) : 26-31.
49. ORTIZ H., DE MIGUEL M., RINALDi M., OTEIZA F., ALTOMARE D.F. Functional outcome of sacral nerve stimulation in patients with severe constipation. Dis. Colon Rectum, 2012, 55(8) : 876-80.

50. RATTO C., GANIO E., NALDINI G. Long-term results following sacral nerve stimulation for chronic constipation. Colorectal disease : the official journal of the Association of Coloproctology of Great Britain and Ireland, $2015,17(4): 320-8$.

51. JANSSEN P.T.J., MEYER Y.M., VAN KUIJK S.M.J., BENNINGA M.A., STASSEN L.P.S., BOUVY N.D., et al. Long-term outcome of intractable constipation treated by sacral neuromodulation: a comparison between children and adults. Colorectal disease : the official journal of the Association of Coloproctology of Great Britain and Ireland, 2018, 20(2) : 134-43

52. GORTAZAR DE LAS CASAS S., RUBIO-PÉREZ I., SAAVEDRA AMBROSY J., SANCHO DE AVILA A., ÁlVAREZ-GALlEGO M., MARIJUAN MARTÍN J.L., et al. Sacral nerve stimulation for constipation long-term outcomes. Tech. Coloproctol., 2019, 23(6) : 559-64.

53. KENEFICK N.J., NICHOLLS R.J., COHEN R.G., KAMM M.A. Permanent sacral nerve stimulation for treatment of idiopathic constipation. Br. J. Surg, 2002, 89(7) : 882-8.

54. KAMM M.A., DUDDING T.C., MELENHORST J., JARRETT M., WANG Z., BUNTZEN S., et al. Sacral nerve stimulation for intractable constipation. Gut, 2010, 59(3) : 333-40

55. SHARMA A., LIU B., WAUDBY P., DUTHIE G.S. Sacral neuromodulation for the management of severe constipation: development of a constipation treatment protocol. International journal of colorectal disease, 2011, 26(12): 1583-7.

56. GRAF W., SONESSON A.C., LINDBERG B., ÅKERUD P., KARLBOM U. Results after sacral nerve stimulation for chronic constipation. Neurogastroenterology and motility : the official journal of the European Gastrointestinal Motility Society, 2015, 27(5) : 734-9.

57. MAEDA Y., KAMM M.A., VAIZEY C.J., MATZEL K.E., JOHANSSON C., ROSEN H., et al. Long-term outcome of sacral neuromodulation for chronic refractory constipation. Tech. Coloproctol., 2017, 21(4) : 277-86.

58. YANG Y., YIM J., CHOI W., LEE S. Improving slow-transit constipation with transcutaneous electrical stimulation in women : A randomized, comparative study. Women Health, 2017, 57(4) : 494-507.

59. MOORE J.S., GIBSON P.R., BURGELL R.E. Randomised clinical trial: transabdominal interferential electrical stimulation vs sham stimulation in women with functional constipation. Aliment. Pharmacol. Ther., 2020 51(8) : 760-9. 